\title{
A note on some type specimens of G. S. Brady's South Sea island ostracods
}

\author{
PETER S. DAVIS \& DAVID J. HORNE \\ Hancock Museum, Newcastle-upon-Tyne NE2 4PT \& Geology Dept, City of London \\ Polytechnic, Walburgh House, Bigland Street, London E1 2NG
}

\begin{abstract}
Provisional registration numbers allocated to some type specimens of ostracods described by G. S. Brady from the SW Pacific are replaced with Hancock Museum Registration Numbers.
\end{abstract}

\section{DISCUSSION}

McKenzie (1986) designated and illustrated type specimens of a number of ostracod species originally described by G. S. Brady (1890) from islands in the SW Pacific. McKenzie originally borrowed the specimens before the recent cataloguing of the Brady Collection (see Davis \& Horne, 1985) had been started, however, and the re-curation of the specimens that he returned to the museum occurred too late for the registration numbers to be included in his paper. The Hancock Museum registration numbers quoted by McKenzie are therefore only provisional ones and are meaningless in the context of the present computer catalogue. This note is intended to set the record straight by presenting the new numbers alongside McKenzie's provisional ones.

To McKenzie's plate and figure numbers we have added the correct Hancock Museum registration number of each specimen, with McKenzie's provisional registration number in brackets. Where McKenzie's generic assignment differs from that originally cited by Brady, both names are given. Further notes are made where appropriate.

\section{Plate 1 (McKenzie, 1986)}

Fig. 8. Cytherelloidea fijiensis $=$ Limnicythere fijiensis Brady, 1890; lectotype carapace, 1.18.28 (B455)

Fig. 9. Cyprideis consobrina $=$ Cytheridea consobrina Brady, 1890; lectotype female carapace, 1.55.23 (B457)

Fig. 10. ?Thalmannia scotti $=$ Cythere scotti Brady, 1890; lectotype carapace, 1.55 .36 (B448).

Fig. 11. ?Pseudaurila guttata $=$ Cytheropteron guttatum Brady, 1890; holotype RV + LV, 1.20.21 (B465).

Fig. 12. Triebelina truncata $=$ Bairdia truncata Brady, 1890 (non Bairdia truncata Kirkby, 1858); lectotype carapace, 1.55 .03 (B441).

Fig. 13. Loxocorniculum marcida $=$ Cytherura marcida Brady, 1890; lectotype carapace, 1.31 .29 (B460).

Fig. 14. Loxoconcha gracilis Brady, 1890: lectotype male carapace, 1.31 .29 (B458).
Fig. 15. Paracytheridea trilobites $=$ Cytheropteron trilobites Brady, 1890; lectotype carapace, 1.55.32 (B467).

Fig. 16. Callistocythere crenata $=$ Cythere crenata Brady, 1890; lectotype carapace, 1.55.25 (B443).

Fig. 17. ?Tanella ochracea $=$ Cythere ochracea Brady, 1890; lectotype carapace, 1.35.39 (B444).

Fig. 18. Paranesidea ventricosa $=$ Bairdia ventricosa Brady, 1890 (non Bairdia ventricosa Kirkby, 1858); lectotype carapace, 1.55 .33 (B440).

Fig. 19. Paracytheridea longicaudata $=$ Cytheropteron longicaudatum Brady, 1890; lectotype carapace (split into LV + RV after return to Hancock Museum), 1.55.39 (B466).

Fig. 20. Ishizakiella inflata $=$ Cythere inflata Brady, 1890; paralectotype female carapace, 1.58 .11 (B445b).

Fig. 21. Paranesidea nodulifera $=$ Bairdia nodulifera Brady, 1890; holotype carapace, 1.33.11 (B442).

Fig. 22. Tenedocythere deltoides $=$ Cythere deltoides Brady, 1890; lectotype carapace, 1.12 .39 (B450).

Fig. 23. Ishizakiella inflata $=$ Cythere inflata Brady, 1890; lectotype male carapace, 1.24.33 (B445a).

Plate 2 (McKenzie, 1986)

Fig. 1. Cytheropteron rude Brady, 1890; lectotype carapace 1.42 .32 (B464).

Fig. 2. Gambiella caudata = Cythere caudata Brady, 1890; lectotype female RV + LV, 1.55.24 (B447a).

Fig. 3. Sarsiella rudis Brady, 1890; lectotype carapace, 1.42.25 (B481).

Fig. 4. Neomonoceratina entomon = Cytherura entomon Brady, 1890; lectotype carapace, 1.15.16 (B461).

Fig. 5. Rutiderma (Alternatochelata) sp. (non Sarsiella sculpta Brady, 1890); specimen presumably separated from Brady's original material of S.sculpta (McKenzie (1986) gives the locality as "off Cap Bon Louis, 4 fathoms, near Noumea", but on the 
slide he has written "Levuka, Fiji"), carapace, 1.55.11 (no number given by McKenzie (1986)).

Fig. 6. Sarsiella foveata Brady, 1890; lectotype carapace, 1.18.21 (B482).

Fig. 7. Hemicytherura scutellata $=$ Cytherura scutellata Brady, 1890; holotype carapace, 1.55 .04 (B462).

Fig. 8. Morkhovenia cuneola $=$ Cythere cuneolus Brady, 1890; lectotype carapace, 1.55.28 (B446).

Fig. 9. Alatahermanites infundibulata $=C y$ there infundibulata Brady, 1890; lectotype RV + LV, 1.24.35 (B451).

Fig. 10. Sarsiella sculpta Brady, 1890; lectotype carapace, 1.55.10 (B479).

Fig. 11. "Cythere" torticollis = Cythere torticollis Brady, 1890; lectotype carapace, 1.55.31 (B449).

Fig. 12. ?Ponticocythereis labiata $=$ Cythere labiata Brady, 1890; holotype carapace, 1.28.44 (B452).

Fig. 13. Ponticocythereis quadriserialis $=$ Cythere quadriserialis Brady, 1890; lectotype carapace, 1.40.10 (B454).

Fig. 14. Ponticocythereis ichthyoderma $=$ Cythere ichthyoderma Brady, 1890; lectotype female carapace, 1.24.32 (B453).

Fig. 15. Streptoleberis crenulata Brady, 1890; lectotype juvenile (B478); after photography by McKenzie this specimen was dissected and illustrated by Kornicker (1978), who determined it to be an adult female; the dissected appendages are on glass slides numbered 1.10.39 and 1.10.40. McKenzie's illustration is of the right side of the carapace; Kornicker also took SEM photographs before softening and dissecting the specimen, illustrating left lateral and anterior views. As Kornicker pointed out, although Brady (1890) stated that he had only a single specimen, two were listed as "types" in Brady's notebook; one of these remains on slide no. 2.14.07 (square 9), while the other, taken from slide 2.14.08, is the lectotype designated by McKenzie.

\section{Text figures (McKenzie, 1986)}

Fig. 3. Gambiella caudata = Cythere caudata Brady, 1890; lectotype female LV, 1.55.24 (B447a).

Fig. 4. Gambiella caudata $=$ Cythere caudata Brady, 1890; paralectotype male RV + LV, 1.58 .12 (B447b).

The following additional type specimens of some of the above species were not mentioned or illustrated by McKenzie (1986):

Cytherelloidea fijienses $=$ Limnicythere fijiensis Brady, 1890; paralectotype carapace, 1.58.07.

Cyprideis consobrina = Cytheridea consobrina Brady, 1890; paralectotype male carapace, 1.58.08.

Loxocorniculum marcida $=$ Cytherura marcida Brady, 1890; paralectotype carapace, 1.58.09.
Loxoconcha gracilis Brady, 1890; paralectotype female carapace, 1.58.10.

Ponticocythereis ichthyoderma $=$ Cythere ichthyoderma Brady, 1890; paralectotype male carapace, 1.58.13.

Manuscript received November 1987

Manuscript accepted November 1987

\section{REFERENCES}

Brady, G. S. 1890 . On Ostracoda collected by H. B. Brady, Esq., L.L.D., F.R.S., in the South Sea Islands. Trans. Roy. Soc. Edinburgh, 35, 489-525.

Davis, P. S. \& Horne, D. J. 1985. George Stewardson Brady (1832-1921) and his collections at the Hancock Museum. Newcastle-upon-Tyne. J. micropalaeontol., 4, 141-152.

Kornicker, L. S. 1978. Harbansus, a new genus of marine Ostracoda, and a revision of the Philomedidae (Myodocopina). Smithsonian Contributions to Zoology, no. 260, 75 pp.

McKenzie, K. G. 1986. A comparative study of collections from the S.W. Pacific (Saipan to Tonga) with the descriptions of Gambiella caudata (Brady, 1890) and a new species of Pterobairdia (Ostracoda). J. micropalaeontol., 5, 91 -108 . 